\title{
Ion-matter interactions by MD simulations making use of reactive force fields
}

\author{
P. Philipp, ${ }^{a *}$ Y. Yue, ${ }^{b}$ T. Wirtz ${ }^{a}$ and J. Kieffer ${ }^{b}$
}

In the field of SIMS, ion-matter interactions have been largely investigated by numerical simulations like TRIM (or other programs using the binary-collision approximation) or molecular dynamics (MD) simulations. For MD simulations related to inorganic samples, mostly classical force fields assuming stable bonding structure have been used.

In materials science, level-three force fields capable of simulating the breaking and formation of chemical bonds have recently been conceived. One such force field has been developed by Kieffer et al ${ }^{[1-4]}$ This potential includes directional covalent bonds, Coulomb and dipolar interaction terms, dispersion terms, etc. Important features of this force field for simulating systems that undergo significant structural reorganization are: (i) the ability to account for the redistribution of electron density upon ionization, formation, or breaking of bonds, through a charge transfer term; and (ii) the fact that the angular constraints dynamically adjust when a change in the coordination number of an atom occurs.

In this work, we will present preliminary results of this potential, parameterized for silicon, for the simulation of atomic trajectories in samples subject to ion bombardment. Compared to normal force fields, ion-matter interactions as well as the sputtering of matter are expected to be described more accurately, especially when using reactive primary ions (oxygen or cesium) at low-impact energies. Copyright (c) 2010 John Wiley \& Sons, Ltd.

Keywords: MD simulations; reactive force field; ion-matter interactions; Si potential; Si(001) surface reconstruction

\section{Introduction}

In SIMS, the interaction of the incoming primary ions with the analyzed sample determines the sputtering of matter as well as atomic mixing and roughness formation at the crater bottom. Consequently, the ion-sample interactions have been largely investigated to understand and control the primary ion-induced damage formation and the related SIMS artefacts to optimize the analysis conditions. Computational studies have been used to explore those different aspects, either by using simulation codes that make use of the binary collision approximation (like for example TRIM) to model the sputtering and defect formation for the irradiation of samples with energetic ions, ${ }^{[5-8]}$ or by using molecular dynamics (MD) simulations to study the atomic-scale mechanisms in ion-matter interactions for both atomic ${ }^{[9-13]}$ or cluster primary ions. ${ }^{[14-23]}$ For the first application, the fragmentation of the sputtered matter and the crater formation under cluster bombardment are of major interest. For inorganic samples, most studies involve the cascade and crater formation. Mainly for this last type of simulations classical force fields have been used, i.e. either covalent or ionic bonding was assumed.

In our group, the optimization of secondary $\mathrm{M}^{-}$and $\mathrm{MCs}_{x}{ }^{+}$ion emission has been studied thoroughly by combining SIMS analyses with the simultaneous deposition of metallic cesium. ${ }^{[2,25]}$ In such systems, a realistic description can only be achieved when partial charge transfers between the deposited $\mathrm{Cs}^{0}$ atoms or the $\mathrm{Cs}^{+}$ ions, and the atoms of the target are considered. To get an insight into the atomic-scale mechanisms during the irradiation of such systems, we develop MD simulations making use of a level-three reactive force field. Such force fields have been developed in materials science for the study of systems undergoing significant structural changes. This technique should not only improve the results for the above systems, but for any system involving the sputtering by reactive species. Overall, the description of the local structure of mixed covalent-ionic systems should be improved. However, no predictions on secondary ion ionization will be possible.

In this paper, we describe preliminary results obtained for the low-energy oxygen bombardment of the Si(001) surface by adapting the code developed by Kieffer et al. ${ }^{[1-4]}$ and we will give an outlook on developments to be performed in the future. The work includes the parameterization of the force field for silicon, the preparation of a $\mathrm{Si}(100)$ surface as well as first results obtained for ion bombardment.

\section{Potential Model}

The force field used in this project was developed by Kieffer et al. to simulate the structure and dynamic properties of compounds with mixed ionic-covalent bonding, such as silica ${ }^{[1,3,26]}$ and hybrid organic-inorganic compounds. ${ }^{[27-30]}$ Important features of this force field are the ability to account for the formation and breaking of bonds. Additionally, the dynamic adjustment of the angular constraints when a change in the coordination number of the atoms occurs is included. It is a general potential that should be able to reproduce the properties of many systems, and not only

Correspondence to: P. Philipp, Department "Science and Analysis of Materials" (SAM), Centre de Recherche Public - Gabriel Lippmann, 41 rue du Brill, L-4422 Belvaux, Luxembourg. E-mail:philipp@lippmann.lu

a Department "Science and Analysis of Materials" (SAM), Centre de Recherche Public - Gabriel Lippmann, 41 rue du Brill, L-4422 Belvaux, Luxembourg

b Department of Materials Science and Engineering, University of Michigan, Ann Arbor, Michigan 48109-2136, USA 
the specific properties of a well defined system. The reactive threebody potential includes a Coulomb term, a Born-Huggins-Mayer repulsive term as well as a three-body term:

$$
\begin{aligned}
\phi_{i}= & q_{i} \sum_{j=1}^{N} \frac{q_{j}}{4 \pi \varepsilon_{0} r_{i j}}+\sum_{j=1}^{N C} C_{i j} e^{\left(\sigma_{i}+\sigma_{j}-r_{i j}\right) \cdot \rho_{i j}} \\
& +\sum_{j=1}^{N C-1} \sum_{k=j+1}^{N C}\left(\varphi_{i j}+\varphi_{i k}\right) e^{-\gamma_{i j k}\left(\bar{\theta}-\theta_{i j k}\right)^{2}}
\end{aligned}
$$

where $\varphi_{i}$ is the potential energy of the particle, $q_{i}$ is the charge, $\varepsilon_{0}$ is the dielectric constant of the vacuum and $r_{i j}$ is the interatomic distance. The charge of each atom is given by equation $q_{i}=q_{i}^{O}-\sum_{j=1}^{N C} \delta_{i j} \zeta_{i j}$, where $q_{i}^{0}$ is the charge of the isolated atom and $\zeta_{i j}=\frac{1}{1+e^{b\left(r_{i j}-a\right)}}$ is the charge transfer function. Both, $a$ and $b$ are empirical parameters. Covalent bonding is modeled by $\left(\varphi_{i j}+\varphi_{i k}\right) e^{-\gamma_{i j k}\left(\bar{\theta}-\theta_{i j k}\right)^{2}}$, where $\varphi_{i j}=-C_{i j} \frac{\kappa_{i j}}{\eta_{i j}} \zeta_{i j} e^{\left(\lambda_{i j}-r_{i j}\right) \eta_{i j}}, \bar{\theta}$ is the equilibrium bond angle and $\theta_{i j k}$ is the angle formed by the bond vectors $r_{i j}$ and $r_{i k}$. Furthermore, $C_{i j}=A_{i j}\left(1+\frac{z_{i}}{n_{i}}+\frac{z_{j}}{n_{j}}\right)$ where $z_{i}$ is the valence and $n_{i}$ is the number of electrons in the outer shell of atom $i$. A more detailed description can be found in Ref. [1]. Compared to classical force fields, the computational cost is increased only slightly and system sizes or time scales are not affected.

\section{Results}

Up to now, only bulk properties and phase transitions in bulk glass systems have been investigated by this three-body potential. The study described in this paper involves the first application of the potential to a system with finite extents in one dimension, i.e. a Si slab with free (001) surfaces.

The parameters for the $\mathrm{Si}-\mathrm{O}$ interactions have already been determined for the simulations on the cristobalite silica. The Si parameters for this reactive potential are reported for the first time in this paper. The values for both the $\mathrm{Si}-\mathrm{O}^{1}$ and $\mathrm{Si}-\mathrm{Si}$

\begin{tabular}{|c|c|c|c|c|c|c|}
\hline \multicolumn{2}{|l|}{ Element } & $\sigma_{i}(\mathrm{~nm})$ & \multicolumn{2}{|r|}{$n_{i}$} & $z_{i}$ & $q_{i}^{0}$ \\
\hline \multicolumn{2}{|l|}{$\mathrm{Si}$} & \multirow[t]{2}{*}{0.1010} & \multicolumn{2}{|r|}{8} & +4 & 0 \\
\hline \multicolumn{2}{|l|}{$\mathrm{O}$} & & \multicolumn{2}{|r|}{8} & -2 & -2 \\
\hline Pair & $\begin{array}{c}A_{i j} \\
\left(10^{-19} \mathrm{~J}\right)\end{array}$ & $\begin{array}{c}\rho_{i j} \\
\left(\mathrm{~nm}^{-1}\right)\end{array}$ & \multicolumn{2}{|l|}{$\begin{array}{c}\lambda_{i j} \\
(\mathrm{~nm})\end{array}$} & $\begin{array}{c}\eta_{i j} \\
\left(\mathrm{~nm}^{-1}\right)\end{array}$ & $\begin{array}{c}\kappa_{i j} \\
\left(\mathrm{~nm}^{-1}\right)\end{array}$ \\
\hline $\mathrm{Si}-\mathrm{Si}$ & 1.19 & 65 & 1.8 & & 1 & 26.4 \\
\hline $\mathrm{Si}-\mathrm{O}$ & 0.18 & 36.7 & 2.6 & & 3.2 & 36.7 \\
\hline $\mathrm{O}-\mathrm{O}$ & 0.25 & 19.5 & 0.0 & & 0.0 & 19.5 \\
\hline \multicolumn{2}{|c|}{ Charge transfer } & $\delta_{i j}(\mathrm{e})$ & \multicolumn{2}{|r|}{$a(n m)$} & \multicolumn{2}{|c|}{$b(n m)$} \\
\hline \multicolumn{2}{|l|}{$\mathrm{Si}$} & 0.0 & \multicolumn{2}{|r|}{0.293} & \multicolumn{2}{|c|}{110} \\
\hline \multicolumn{2}{|l|}{$\mathrm{O}$} & -0.2830 & \multicolumn{2}{|r|}{0.240} & \multicolumn{2}{|c|}{80} \\
\hline \multicolumn{2}{|l|}{ Triplet } & $\gamma_{i j k}\left(\operatorname{rad}^{-2}\right)$ & \multicolumn{3}{|c|}{$\theta(\mathrm{rad})$} & \\
\hline \multicolumn{2}{|l|}{$\mathrm{Si}-\mathrm{Si}-\mathrm{Si}$} & 0.28 & \multicolumn{3}{|c|}{1.91} & \\
\hline \multicolumn{2}{|c|}{$\mathrm{O}-\mathrm{Si}-\mathrm{O}$} & 0.20 & \multicolumn{3}{|c|}{1.91} & \\
\hline \multicolumn{2}{|l|}{$\mathrm{Si}-\mathrm{O}-\mathrm{Si}$} & 0.30 & \multicolumn{3}{|c|}{2.48} & \\
\hline
\end{tabular}

Table 1. Parameters for the $\mathrm{Si}-\mathrm{Si}$ and $\mathrm{Si}-\mathrm{O}$ interactions

interactions are given in Table 1. The parameters for silicon have been optimized by reproducing the phonon density of states (Fig. 1(a)). The highest frequency peak at $520 \mathrm{~cm}^{-1}$ is also observed experimentally by Raman spectroscopy. The frequencies of this and the remaining peaks were obtained either by $a b$ inito calculations $^{[31]}$ or by IR spectroscopy on amorphous silicon. ${ }^{[32]}$ For the three peaks with the highest frequencies, our data agrees well with the values from literature. The differences in frequency between our data and the $a b$ inito calculations do not exceed $15 \mathrm{~cm}^{-1}$, while the differences between our data and the IR spectra are only slightly higher $\left(\max .25 \mathrm{~cm}^{-1}\right)$. For the lowestfrequency peak, the values range from $140 \mathrm{~cm}^{-1}$ for the IR spectra up to $220 \mathrm{~cm}^{-1}$ for the peak obtained by $a b$ inito calculations. Our peak lies in between at $180 \mathrm{~cm}^{-1}$.

In Fig. 1(b)), the form of our potential is compared to classical force fields parameterized for $\mathrm{Si} .{ }^{[33]}$ Our potential shows the largest curvature in the potential well, only in this way the vibrational
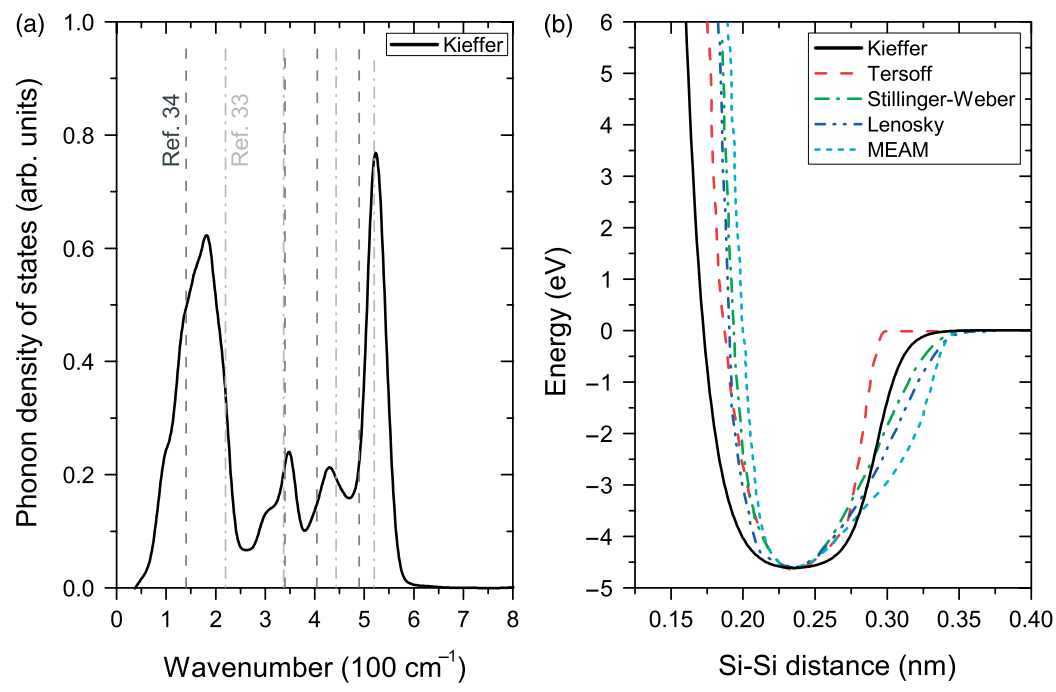

Figure 1. Phonon density of states for our parameter set, a) and comparison of different potential forms, b). 

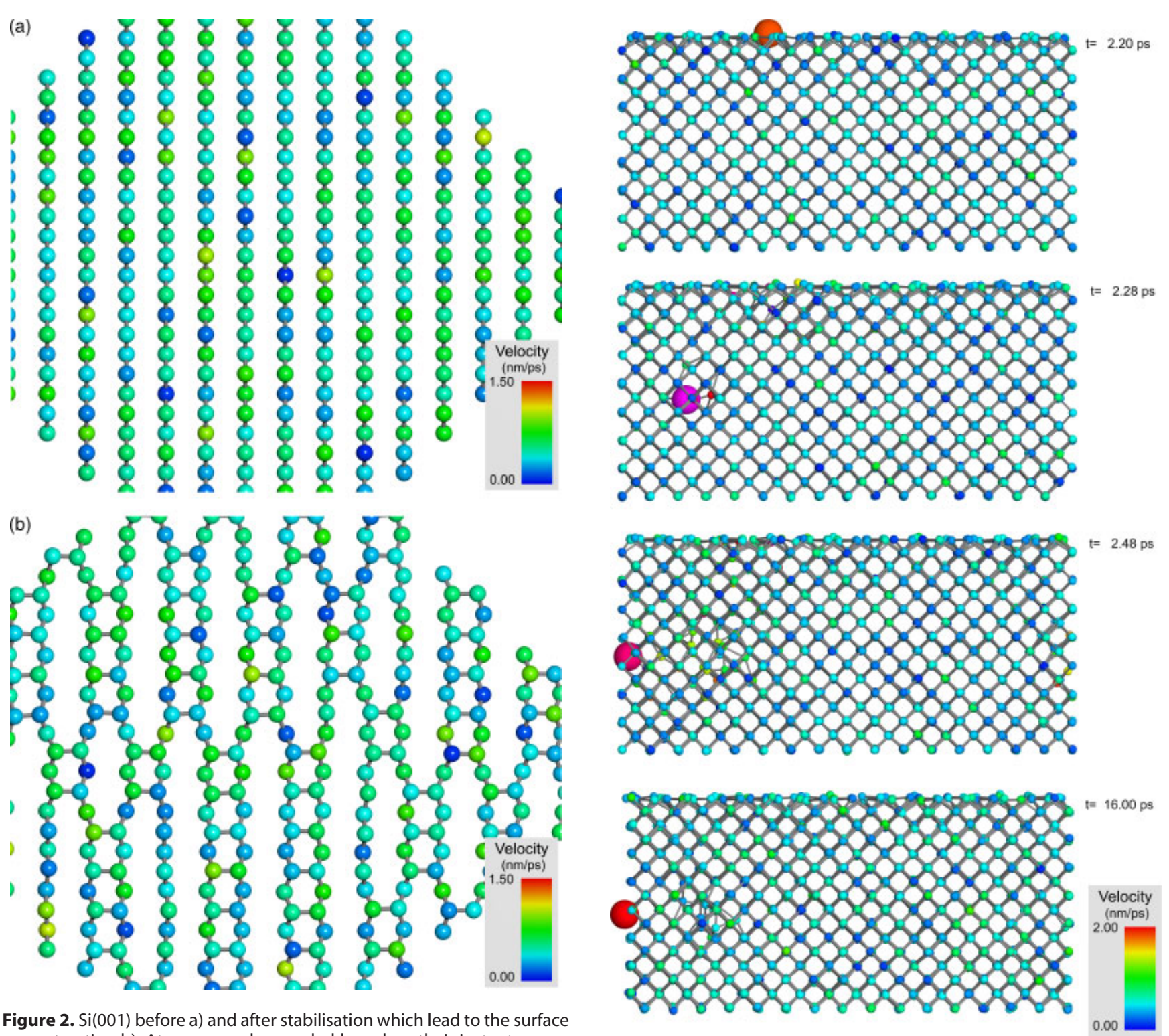

Figure 2. Si(001) before a) and after stabilisation which lead to the surface reconstruction b). Atoms are colour-coded based on their instantaneous velocities.

modes of Si could not be reproduced. The repulsive parts of the different potentials are similar (same steepness) while the cut-off distance of our potential lies in between the Tersoff and the Stillinger-Weber potential.

In addition to the bulk properties, the potential must be able to reproduce surface properties, like for example, the surface reconstruction of the $\mathrm{Si}(001)$ surface. Figure 2 shows the $\mathrm{Si}(001)$ surface before stabilization and after a simulation time of $100 \mathrm{ps}$ at $300 \mathrm{~K}$ for a system containing 8000 atoms (crystal dimensions of $5.43 \times 5.43 \times 5.43 \mathrm{~nm}^{3}$ ). In Fig. 3(b)), the Si-Si dimer bonds form different domains on the stabilized surface. This is also observed experimentally and has already been reproduced in MD simulations by some classical force fields.

These results are important for the overall quality of the parameters set for silicon to describe this material as realistically as possible, but they do not lead automatically to a good description of the sputtering process. This is also true for the $\mathrm{Si}-\mathrm{O}$ interactions. For the oxygen bombardment of the Si(001) surface, only preliminary results can be reported here. Figure 3 shows

Figure 3. $100 \mathrm{eV}$ oxygen on $\mathrm{Si}(001)$ with an incidence angle of $80^{\circ}$. Atoms are colour-coded based on their instantaneous velocities.

defect formation in silicon under $100 \mathrm{eV}$ oxygen bombardment, at an incidence angle of $80^{\circ}$. However, this particular impact does not lead to the ejection of matter. At such low-impact energies, the sputter yield under oxygen bombardment is low. Conclusive results can only be obtained by a significantly larger number of impacts. Simulations to this effect are currently underway.

\section{Conclusion}

A simulation code making use of a reactive force field and developed for the study of structural and dynamic properties of bulk systems has been modified for the simulation of ion bombardment. The main objective is to obtain an improved description of ion-matter interactions under low-energy bombardment involving reactive species. The preliminary results show that, in addition to the other systems previously investigated using this force field, the potential is now successfully parameterized for silicon. Pa- 
rameter optimization is based on reproducing the bulk structure and the phonon density of states of Si. Subsequently, during the equilibration of a thin silicon slab, the experimentally observed surface reconstruction on $\mathrm{Si}(001)$ is reproduced naturally. However, no conclusive results for the bombardment of $\mathrm{Si}(001)$ by $100 \mathrm{eV}$ oxygen have been obtained for lack of statistics. A larger number of collision cascades need to be examined.

Various bombardment conditions will be explored. Furthermore, a more realistic description of the ion-matter interactions, in particular, defect formation, should become possible when using the coordinate-dependent version of the force field, which has already been applied successfully to the simulation of several systems in materials science.

\section{References}

[1] L. Huang, J. Kieffer, J. Chem. Phys. 2003, 118, 1487.

[2] T. C. lonescu, F. Qi, C. McCabe, A. Striolo, J. Kieffer, P. T. Cummings, J. Phys. Chem. B 2006, 110, 2502.

[3] L. P. Huang, M. Durandurdu, J. Kieffer, Nat. Mater. 2006, 5, 977.

[4] L. Huang, J. Kieffer, Phys. Rev. B 2006, 74, 224107.

[5] J. P. Biersack, L. G. Haggmark, Nucl. Instrum. Methods 1980, 174, 257.

[6] W. Moller, W. Eckstein, Nucl. Instrum. Methods Phys. Res. B 1984, 230, 814.

[7] V. I. Shulga, W. Eckstein, Nucl. Instrum. Methods Phys. Res. B 1998 $145,492$.

[8] W. Eckstein, V. I. Shulga, Nucl. Instrum. Methods Phys. Res. B 2000, $164-165,748$.

[9] I. Koponen, M. Hautala, O. P. Sievänen, Nucl. Instrum. Methods Phys. Res. B 1997, 127-128, 230.

[10] K. Gärtner, B. Weber, Nucl. Instrum. Methods Phys. Res. B 2001, 180, 274.

[11] R. Smith, D. E. Harrison, B. J. Garrison, Phys. Rev. B 1989, $40,93$.
[12] R. Aderjan, H. M. Urbassek, Nucl. Instrum. Methods Phys. Res. B 2000, $164-165,697$.

[13] R. Pinzon, H. M. Urbassek, Phys. Rev. B 2001, 63, 195319.

[14] A. Delcorte, B. J. Garrison, Nucl. Instrum. Methods Phys. Res. B 2007, 255, 223.

[15] E. J. Smiley, N. Winograd, B. J. Garrison, Anal. Chem. 2007, 79, 494.

[16] M. F. Russo, Z. Postawa, B. J. Garrison, J. Phys. Chem. C 2009, 113, 3270.

[17] T. C. Colla, H. M. Urbassek, Nucl. Instrum. Methods Phys. Res. B 2000, $164-164,687$.

[18] C. Anders, H. M. Urbassek, Nucl. Instrum. Methods Phys. Res. B 2005, $228,57$.

[19] C. Anders, H. Kirihata, Y. Yamaguchi, H. M. Urbassek, Nucl. Instrum. Methods Phys. Res. B 2007, 255, 247.

[20] K. Nordlund, T. T. Jarvi, K. Meinander, J.Samela, Appl. Phys. A-Materials Science \& Processing 2008, 91, 561.

[21] K. Nordlund, M. Ghaly, R. S. Averback, M. Caturla, T. D. de la Rubia, J. Tarus, Phys. Rev. B 1998, 57, 7556.

[22] K. O. E. Henriksson, K. Nordlund, J. Keinonen, Nucl. Instrum. Methods Phys. Res. B 2007, 255, 259.

[23] J. Samela, K. Nordlund, Nucl. Instrum. Methods Phys. Res. B 2007, 263, 375.

[24] T. Wirtz, H. N. Migeon, Surf. Sci. 2004, 557, 57.

[25] P. Philipp, T. Wirtz, H. N. Migeon, H. Scherrer, Int. J. Mass Spectrom. 2006, 253, 71 .

[26] L. P. Huang, J. Kieffer, Phys. Rev. B 2004, 69, 224203.

[27] S. Bhattacharya, J. Kieffer, J. Chem. Phys. 2005, 122, 094715.

[28] L. P. Huang, M. Durandurdu, J. Kieffer, J. Phys. Chem. C 2007, 111, 13712.

[29] S. Bhattacharya, J. Kieffer, J. Phys. Chem. C 2008, 112, 1764.

[30] J. H. Zhou, J. Kieffer, J. Phys. Chem. C 2008, 112, 3473.

[31] J. Y. Chen, J. F. Vetelino, S. S. Mitra, Solid State Commun. 1975, 16, 1313.

[32] M. H. Brodsky, A. Lurio, Phys. Rev. B 1974, 9, 1646.

[33] B. J. Thijsse, T. P. C. Klaver, E. F. C. Haddeman, Appl. Surf. Sci. 2004, $231-232,29$. 